\title{
A RIEMANN TYPE THEOREM FOR UNCONDITIONAL CONVERGENCE OF OPERATORS
}

\author{
VICTOR KAFTAL AND GARY WEISS ${ }^{1}$
}

\begin{abstract}
We prove that if a series of bounded linear operators is compactly conditionally convergent in the strong operator topology, that is, each of its partial sums converge, in the strong operator topology to a compact operator, then the series converges in the uniform (operator norm) topology; although not necessarily absolutely. In case the operators are all mutually diagonalizable, then under the same hypothesis, the series converges absolutely uniformly.
\end{abstract}

1. Introduction. The classical Riemann theorem states that if a series of real (or complex) numbers converges unconditionally, then it converges absolutely [4, Theorem 3.56].

This paper studies a generalization of the Riemann theorem to series of bounded operators on a Hilbert space.

The S.O.T. unconditional convergence (sometimes called the commutative convergence) of a series of bounded operators is defined as the convergence in the S.O.T. (the strong operator topology) of the net of the finite partial sums (see Definition 1).

The simple example of a series $\sum_{n=1}^{\infty} p_{n}$ of mutually orthogonal projections $p_{n}$ shows that the usual absolute convergence cannot be deduced from the unconditional convergence of a series.

We shall investigate here series whose partial sums (which converge unconditionally in the S.O.T.) are compact. The usual notion of absolute convergence of a series in a normed space, namely that the series of the norms converges, is much too strong to be linked to unconditional convergence even with this additional condition, as we see by considering $\sum_{n=1}^{\infty} n^{-1} p_{n}$ where the $p_{n}$ are mutually orthogonal finite projections.

In Theorem 2 we prove that if all the partial sums are compact then the series converges uniformly. In Proposition 6 we obtain that if all the operators are diagonal and satisfy the above condition, then the series of the absolute values of the operators converges uniformly $\left(|T|=\left(T^{*} T\right)^{1 / 2}\right.$ is the absolute value of the operator $T)$. Examples 4 and 5 show that we cannot relax these conditions.

Our interest in such questions arose from the following problem connected to our work on relative compact derivations [2]: given an atomic masa $A$ of a von

Received by the editors October 31, 1985.

1980 Mathematics Subject Classification (1985 Revision). Primary 47B05, $40 \mathrm{~J} 05$.

${ }^{1}$ Partially supported by N.S.F. Grant DMS-8503390. 
Neumann algebra $M$ and a derivation $\delta$ from $A$ into an ideal $J$ of $M$, can $\delta$ be extended to a derivation $\tilde{\delta}$ from the algebra generated by $A$ and the center of $M$ into $J$ ? The values of $\tilde{\delta}$ are sums of unconditionally convergent series with entries from $J$.

We wish to thank M. D. Choi for a useful discussion and the idea that lead us to Example 5.

\section{The main result.}

DEFINITION 1. Let $\left\{K_{n}\right\}$ be a sequence of bounded linear operators on a Hilbert space $H$ and let $F$ denote any subset of the set of positive integers $\mathbf{N}$. Then we say that the series $\sum_{n \in F} K_{n}$ converges unconditionally in the S.O.T. if the net of the finite partial sums converges in the S.O.T. to a bounded operator $K$, i.e., if for every $\xi \in H$ and $\varepsilon>0$ there is a finite subset $E$ of $F$ such that for all finite subsets $G$ of $F$ with $E \subset G$ we have $\left\|\left(K-\sum_{n \in G} K_{n}\right) \xi\right\|<\varepsilon$.

This is equivalent to ask that all rearrangements of the series converge in the S.O.T. to $K[1,5.3]$. Throughout this paper the symbol $\sum_{n \in F} K_{n}$ will always denote a series unconditionally convergent in the S.O.T. Thus we shall distinguish between $\sum_{n \in \mathrm{N}} K_{n}$ and $\sum_{n=1}^{\infty} K_{n}$ where the latter is the ordinary limit (in the S.O.T.) of $\sum_{j=1}^{n} K_{j}$. We shall henceforth omit the reference to the topology.

Notice that if a series converges unconditionally then the net of finite partial sums is norm bounded. Because of the completeness of the unit ball of $B(H)$ in the S.O.T., the Cauchy condition is necessary and sufficient for unconditional convergence [3, 6.20-23]. Thus $\sum_{n \in F} K_{n}$ converges unconditionally iff for every $\xi \in H$ and $\varepsilon>0$ there is a finite subset $E \subset F$ such that for all finite subsets $G \subset F \backslash E$ we have $\left\|\left(\sum_{n \in G} K_{n}\right) \xi\right\|<\varepsilon$.

As a consequence, we see that any subseries of an unconditionally convergent series is also unconditionally convergent. Also, if $K=\sum_{n \in F} K_{n}, F=\bigcup\{F(m) \mid m=$ $1,2, \ldots\}$ where the $F(m)$ are disjoint sets and $S(m)=\sum_{n \in F(m)} K_{n}$ (unconditionally, by the above remark), then it is easy to verify that $K=\sum_{m \in \mathrm{N}} S(m)$. In particular, $S(m) \rightarrow 0$ in the S.O.T. Also denote by $K(H)$ the compact operators on $H$.

THEOREM 2. Let $K_{n} \in B(H)$ be such that $\sum_{n \in \mathrm{N}} K_{n}$ converges unconditionally and $\sum_{n \in F} K_{n} \in K(H)$ for every index set $F \subset \mathbf{N}$. Then $\sum_{n=1}^{\infty} K_{n}$ converges uniformly.

Proof. Assume not. Then there is an $\varepsilon>0$ and a sequence of finite disjoint index sets $F(m) \subset \mathbf{N} \cap(m, \infty)$, such that $\left\|\sum_{n \in F(m)} K_{n}\right\|>\varepsilon$ for $m=1,2, \ldots$ Let $S(m)$ $=\sum_{n \in F(m)} K_{n}$; by a preceding remark, $S(m) \rightarrow 0$ (S.O.T.) and hence by the uniform boundedness theorem $\|S(m)\|$ is bounded. Without loss of generality we can assume that $\varepsilon<\|S(m)\|<1$. By hypothesis each $S(m)$ is compact, therefore we can find for the operators $S(m)^{*} S(m)$ unit eigenvectors $\eta_{m}$ corresponding to the eigenvalues $\|S(m)\|^{2}$. Let $\varepsilon_{k}=\varepsilon^{2} 2^{-k}, m_{1}=1$ and let $\xi_{1}=\eta_{m_{1}}$; since $S(m) \rightarrow 0$ (S.O.T.) we choose an integer $m_{2}$ such that $\left\|S\left(m_{2}\right) \xi_{1}\right\|<\varepsilon_{2}$. Iterating we find $m_{k}$, $T_{k}=S\left(m_{k}\right), \xi_{k}=\eta_{m_{k}}$ such that $\left\|T_{k} \xi_{j}\right\|<\varepsilon_{k}$ for $j=1,2, \ldots, k-1$. Then for $k>j$ 
we have

$$
\left(\xi_{k}, \xi_{j}\right)=\left\|T_{k}\right\|^{-2}\left(T_{k}^{*} T_{k} \xi_{k}, \xi_{j}\right)=\left\|T_{k}\right\|^{-2}\left(T_{k} \xi_{k}, T_{k} \xi_{j}\right)
$$

whence $\left|\left(\xi_{k}, \xi_{j}\right)\right|<\varepsilon^{-2} \varepsilon_{k}=2^{-k}$. By applying the Gram-Schmidt process to the vectors $\xi_{k}$, we can construct an orthonormal sequence $\zeta_{k}$ and by computing the Gram-Schmidt determinant we can show that $\left\|\xi_{k}-\zeta_{k}\right\| \rightarrow 0$. This shows that $\xi_{k} \rightarrow 0$ in the weak topology. By the Cauchy condition for $\sum_{n \in \mathrm{N}} K_{n}$, for every $\nu \in H$ and $\varepsilon>0$, there is a function $m=m(\nu, \varepsilon / 3)$ such that $\left\|\left(\sum_{n \in G \cap(m, \infty)} K_{n}\right) \nu\right\|$ $<\varepsilon / 3$ for every finite (and hence infinite) subset $G \subset \mathbf{N}$. Let $k_{1}=1, \nu_{1}=\xi_{k_{1}}$ and $\bar{T}_{1}=T_{1}$. Choose $k_{2}>k_{1}$ and $\nu_{2}=\xi_{k_{2}}$ such that

(i) $\inf \bigcup\left\{F\left(m_{k}\right) \mid k \geqslant k_{2}\right\}>m\left(\nu_{1}, \varepsilon / 3\right)$,

(ii) $\left\|\bar{T}_{1} \nu_{2}\right\|<\varepsilon / 3$.

Condition (i) can be satisfied because the sets $F\left(m_{k}\right)$ are finite and disjoint, condition (ii) because $\xi_{k}$ converges to 0 (weakly) and $\bar{T}_{1}$ is compact. Let $\bar{T}_{2}=T_{k_{2}}$. Iterating, construct subsequences $k_{n}, \nu_{n}=\xi_{k_{n}}, \bar{T}_{n}=T_{k_{n}}$ with

(i) $\inf \bigcup\left\{F\left(m_{k}\right) \mid k \geqslant k_{n}\right\}>m\left(\nu_{n-1}, \varepsilon / 3\right)$,

(ii) $\left\|\left(\sum_{k=1}^{n-1} \bar{T}_{k}\right) \nu_{n}\right\|<\varepsilon / 3$.

Let $G_{n}=\bigcup\left\{F\left(m_{k_{1}}\right) \mid i>n\right\}$, and let $\bar{T}=\sum_{i \in G_{0}} K_{i}$. Then $\bar{T}=\sum_{k=1}^{\infty} \bar{T}_{k}$. Similarly, $\sum_{i \in G_{n}} K_{i}=\sum_{k=n+1}^{\infty} \bar{T}_{k}^{\prime}$ and since $G_{n} \subset\left(m\left(\nu_{n}, \varepsilon / 3\right), \infty\right)$, we have $\left\|\sum_{k=n+1}^{\infty} \bar{T}_{k} \nu_{n}\right\|<\varepsilon / 3$. Now

$$
\bar{T} \nu_{n}=\sum_{k=1}^{n-1} \bar{T}_{k} \nu_{n}+\bar{T}_{n} \nu_{n}+\sum_{k=n+1}^{\infty} \bar{T}_{k} \nu_{n}
$$

and by construction the first and third terms have norms less than $\varepsilon / 3$ and the second term has norm larger than $\varepsilon$. Thus we see that $\left\|\bar{T} \nu_{n}\right\|$ does not converge to 0 . But given that $\nu_{n} \rightarrow 0$ weakly, this implies that $\bar{T}$ is not in $K(H)$, against the hypothesis.

The same proof yields the corollary:

COROllaRY 3. Let $K_{n} \in B(H)$ be such that $\sum_{n \in F} K_{n} \in K(H)$ for every index set $F \subset \mathbf{N}$. Then for every $\varepsilon>0$ there is an integer $m$ such that $\left\|\Sigma_{n \in G \cap(m, \infty)} K_{n}\right\|<\varepsilon$ for every index set $G \subset \mathbf{N}$.

The following example shows that we cannot weaken the hypothesis of Theorem 2 and ask only that $\sum_{n \in \mathrm{N}} K_{n} \in K(H)$.

EXAMPLE 4. Let $\left\{p_{n} \mid n=1,2, \ldots\right\}$ be a sequence of mutually orthogonal finite projections. Let $K_{2 n}=p_{n}$ and $K_{2 n-1}=-p_{n}$. Then it is easy to verify that $\sum_{n \in \mathrm{N}} K_{n}$ converges unconditionally to $0 \in K(H)$. but $\Sigma_{n \in \mathrm{N}} K_{2 n}$ is not compact and the conclusion of Theorem 2 is not satisfied.

3. Convergence of the absolute values. While for the real numbers unconditional convergence is equivalent to absolute convergence, i.e. convergence of the series of the norms, we have seen in the introduction that this is no longer true in $B(H)$. We can ask however the weaker question, whether for every unconditionally convergent series of operators $K_{n}$ such that all the partial sums are compact it follows that the 
series of the absolute values $\left|K_{n}\right|$ converges. The answer is in general negative (Example 5), but if the operators are diagonal then the answer is affirmative (Proposition 6).

ExAmple 5. Let $A(n, j)=A(n, j)^{*}$ be the $n \times n$ matrix having 1 in the $(1, j)$ and $(j, 1)$ positions and 0 elsewhere. Then $|A(n, j)|$ is the diagonal matrix having 1 in the $(1,1)$ and $(j, j)$ positions and 0 elsewhere. Thus $\left\|\sum_{j=1}^{k}|A(n, j)|\right\|=k$. On the other hand it is easy to compute $\left\|\sum_{j=1}^{k} A(n, j)\right\|=k^{1 / 2}$. Let $H=\sum_{n=1}^{\infty} \oplus H_{n}$ where $\operatorname{dim} H_{n}=n$ and embed $B\left(H_{n}\right)$ in $B(H)$. Consider then $\sum\left\{n^{-3 / 4} A(n, j) \mid j=\right.$ $1,2, \ldots, n ; n=1,2, \ldots\}$. We see that the series does not converge absolutely, indeed the series of the absolute values "is a positive unbounded operator", i.e. a direct sum of terms with norm $n^{1 / 4}$. On the other hand, it is easy to verify that the series itself, being the direct sum of finite blocks having norm $n^{-1 / 4}$, satisfies the conditions of Theorem 2.

A central phenomenon to this example is the noncommutativity in $B\left(H_{n}\right)$ of the operators $A(n, j)$.

Let us now consider the case of diagonal compact operators $D_{n}$ (relative to a basis $\left\{\zeta_{n}\right\}$ ) satisfying the conditions of Theorem 2 .

Proposition 6. Let $D_{n}$ be a sequence of diagonal compact operators such that $\sum_{n \in \mathrm{N}} D_{n}$ converges unconditionally and $\sum_{n \in F} D_{n} \in K(H)$ for every index set $F \subset \mathbf{N}$, then $\sum_{n=1}^{\infty}\left|D_{n}\right|$ converges uniformly in $K(H)$.

Proof. Without loss of generality we can assume that all $D_{n}$ are selfadjoint, by passing if necessary to the series of $\operatorname{Re}\left(D_{n}\right)$ and $\operatorname{Im}\left(D_{n}\right)$. Let us first notice that since $\sum_{n=1}^{\infty}\left(D_{n} \zeta_{k}, \zeta_{k}\right)$ converges unconditionally, the Riemann theorem applies and hence $\sum_{n=1}^{\infty}\left|\left(D_{n} \zeta_{k}, \zeta_{k}\right)\right|<\infty$ for all $k$. Clearly, $\lim _{k} \sum_{n=1}^{\infty}\left|\left(D_{n} \zeta_{k}, \zeta_{k}\right)\right|=0$ iff the diagonal operator $\sum_{n=1}^{\infty}\left|D_{n}\right|$ is compact iff $\sum_{n=1}^{\infty}\left|D_{n}\right|$ converges uniformly (to a compact operator), where the latter equivalence follows from Theorem 2 and from the fact that here $\sum_{n=1}^{\infty}\left|D_{n}\right|=\sum_{n \in \mathrm{N}}\left|D_{n}\right|$, and hence all partial sums are compact. Thus reasoning by contradiction, assume that $\sum_{n=1}^{\infty}\left|\left(D_{n} \zeta_{k}, \zeta_{k}\right)\right|>\varepsilon$ for some $\varepsilon>0$ and for infinitely many $k$. Construct subsequences of vectors $\nu_{i}=\zeta_{k_{i}}$ and of integers $n_{i}$ such that

(i) $\sum\left\{\mid\left(D_{n} \nu_{i}, \nu_{i}\right) \| n<n_{i-1}\right\}<\varepsilon / 16$,

(ii) $\sum\left\{\mid\left(D_{n} \nu_{i}, \nu_{i}\right) \| n_{i-1} \leqslant n<n_{i}\right\}>\varepsilon / 2$,

(iii) $\sum\left\{\mid\left(D_{n} \nu_{i}, \nu_{i}\right) \| n_{i} \leqslant n\right\}<\varepsilon / 16$.

(i) is obtained from the fact that $\sum\left\{\left|D_{n}\right| \mid n<n_{i-1}\right\} \in K(H)$ and $\zeta_{k} \rightarrow 0$ (weakly), (ii) follows from the hypothesis that $\sum_{n=1}^{\infty}\left|\left(D_{n} \zeta_{k}, \zeta_{k}\right)\right|>\varepsilon$ for infinitely many indices $k$ and (iii) from the fact that $\sum_{n=1}^{\infty}\left|\left(D_{n} \zeta_{k}, \zeta_{k}\right)\right|<\infty$ for all $k$. Being selfadjoint, $D_{n}$ splits into its positive and negative parts $D_{n}=D_{n}^{+}-D_{n}^{+}$, hence

$$
\left|\left(D_{n} \nu_{i}, \nu_{i}\right)\right|=\left(D_{n}^{+} \nu_{i}, \nu_{i}\right)+\left(D_{n}^{-} \nu_{i}, \nu_{i}\right)=\left(D_{n} \nu_{i}, \nu_{i}\right)^{+}+\left(D_{n} \nu_{i}, \nu_{i}\right)^{-}
$$

and therefore

$$
\max \left\{\sum\left\{\left(D_{n}^{+} \nu_{i}, \nu_{i}\right) \mid n_{i-1} \leqslant n<n_{i}\right\}, \sum\left\{\left(D_{n}^{-} \nu_{i}, \nu_{i}\right) \mid n_{i-1} \leqslant n<n_{i}\right\}\right\}>\varepsilon / 4
$$


Without loss of generality we can assume that for infinitely many indices $i_{m}$ the maximum is attained by the positive parts. Let $\xi_{m}=\nu_{i_{m}}$, let $G_{m}=\left\{n \in \mathbf{N} \mid n_{i_{m-1}} \leqslant\right.$ $\left.n<n_{i_{m}},\left(D_{n}^{+} \xi_{m}, \xi_{m}\right)=\left(D_{n} \xi_{m}, \xi_{m}\right)\right\}$ and let $G=\bigcup G_{m}$. Then we have

$$
\begin{aligned}
\left|\left(\left(\sum_{n \in G} D_{n}\right) \xi_{m}, \xi_{m}\right)\right| & \geqslant\left|\sum_{n \in G_{m}}\left(D_{n} \xi_{m}, \xi_{m}\right)\right|-\left|\sum_{k \neq m}\left(\sum_{n \in G_{k}}\left(D_{n} \xi_{m}, \xi_{m}\right)\right)\right| \\
& \geqslant \sum_{n \in G_{m}}\left(D_{n}^{+} \xi_{m}, \xi_{m}\right)-\sum\left\{\left|\left(D_{n} \xi_{m}, \xi_{m}\right)\right| \mid n<n_{i_{m}-1}, n \geqslant n_{i_{m}}\right\} \\
& >\frac{\varepsilon}{4}-\frac{\varepsilon}{8}=\frac{\varepsilon}{8}
\end{aligned}
$$

Since $\xi_{m}$ is orthonormal, this contradicts the assumption that $\sum_{n \in G} D_{n} \in K(H)$ for every set $G$ and thus completes the proof.

Clearly the condition in the above proposition is also sufficient. By similar methods we also obtain

Proposition 7. Let $D_{n}$ be a sequence of diagonal operators such that $\sum_{n \in F} D_{n}$ converges unconditionally to a bounded operator for every index set $F \subset \mathbf{N}$. Then $\sum_{n=1}^{\infty}\left|D_{n}\right|$ converges in the S.O.T. to a bounded operator.

Propositions 6 and 7 hold true trivially replacing S.O.T. by W.O.T. It remains an open question whether or not Theorem 2 can be strengthened by replacing S.O.T. by W.O.T.

ADDED IN PROOF. We proved that the question at the end of the introduction has an affirmative answer.

\section{REFERENCES}

1. J. Dieudonné, Foundations of modern analysis, vol. 10-I, Academic Press, New York, 1969.

2. V. Kaftal and G. Weiss, Compact derivations relative to semifinite von Neumann algebras, J. Funct. Anal. 62 (1985), 202-220.

3. J. Kelley, General topology, Van Nostrand, Princeton, N. J., 1955.

4. W. Rudin, Principles of mathematical analysis, McGraw-Hill, New York, 1953.

Department of Mathematical Sciences, University of Cincinnati, Cincinnati, Ohio 45221 